\title{
Further mechanistic evidence against luminal esophageal temperature monitoring?
}

\author{
James Daniels ${ }^{1}$ and Erik Kulstad ${ }^{1}$ \\ ${ }^{1}$ The University of Texas Southwestern Medical Center
}

February 28, 2021

\begin{abstract}
We congratulate Kar et al. on their elegant study evaluating ex-vivo temperature profiles and the resulting thermal injury formation on the epiesophageal surface during radiofrequency ( $R F$ ) ablation. In addition to being the first study to detail temperature profiles inclusive of the epiesophageal surface during RF ablation, we believe that the results add further concern to the use of temperature sensing technology in the quest to reduce esophageal injury. Three recent clinical trials have evaluated the efficacy of luminal esophageal temperature (LET) monitoring and found either no benefits, or signals of harm. On the other hand, two pilot RCTs suggest benefits of active cooling, and a large RCT, the IMPACT study, further confirms this benefit by finding an $83 \%$ reduction in esophageal lesion formation using an active cooling device. With no degradation in ablation efficacy, as well as a reduction/elimination of the need for fluoroscopy and reports of shortened procedure time with active cooling technology, the data of Kar et al., combined with growing clinical data, suggest that continued use of LET monitoring may be unjustified.
\end{abstract}

\section{Further mechanistic evidence against luminal esophageal temperature monitoring?}

James Daniels, MD, Department of Internal Medicine, Cardiology, UT Southwestern Medical Center, 5323 Harry Hines Blvd., Dallas, TX 75390, James.Daniels@utsouthwestern.edu

Erik Kulstad, MD, MS, Dept. of Emergency Medicine, UT Southwestern Medical Center, 5323 Harry Hines Blvd., Dallas, TX 75390, Erik.Kulstad@UTSouthwestern.edu

Corresponding author: Erik Kulstad, MD, MS, Dept. of Emergency Medicine, UT Southwestern Medical Center, 5323 Harry Hines Blvd., Dallas, TX 75390, Erik.Kulstad@UTSouthwestern.edu

Funding: None

Conflict of interest: EK declares equity interest in Attune Medical.

We congratulate Kar et al. on their elegant and insightful study evaluating ex-vivo temperature profiles and the resulting thermal injury formation on the epiesophageal surface during radiofrequency (RF) ablation.[1] In addition to being the first study to detail temperature profiles inclusive of the epiesophageal surface during $\mathrm{RF}$ ablation, we believe that the results add further concern to the use of temperature sensing technology in the quest to reduce esophageal injury.

Three recent clinical trials have evaluated the efficacy of luminal esophageal temperature (LET) monitoring. The OPERA study, a randomized controlled trial (RCT), demonstrated trends towards greater esophageal lesion formation with the use of single-sensor LET monitoring when ablating with standard power.[2] The AI-HP ESO II study demonstrated trends towards greater esophageal lesion formation with multi-sensor LET monitoring when ablating with ablation-index guided high-power.[3] Most recently, an RCT by Grosse 
Meininghaus et al. found a 14\% injury rate using multi-sensor LET monitoring versus a $5 \%$ rate with no LET monitoring (although the most severe lesion, a deep ulcer, was found in the unmonitored group).[4]

Current recommendations only provide a Class IIa recommendation for LET monitoring, with level of evidence C-EO (expert opinion), and have not incorporated the latest data from these 3 studies demonstrating the lack of benefit, and potential harm, from LET monitoring.[5] Kar et al. found a lag time between the peak epiesophageal and endoluminal temperatures of $13.0 \pm 11.0$ seconds in standard ablation, and $24.2 \pm 22.1$ seconds in high-power, short-duration (HPSD) ablation, with a gradient between peak epiesophageal surface temperature and concurrent endoluminal temperature of $1.7 \pm 2.0{ }^{\circ} \mathrm{C}$ in standard ablation and $5.1 \pm 5.3{ }^{\circ} \mathrm{C}$ in HPSD ablation. Thus, a mechanistic rationale for why LET monitoring has not been shown to provide clinical benefit may be the inability of any measurement technology to identify thermal insult before it has occurred.

On the other hand, two pilot RCTs suggest benefits of active cooling, and a large RCT, the IMPACT study, further confirms this benefit by finding an $83 \%$ reduction in esophageal lesion formation using an active cooling device.[6-8] With no degradation in ablation efficacy, as well as a reduction/elimination of the need for fluoroscopy and reports of shortened procedure time with active cooling technology, the data of Kar et al., combined with growing clinical data, suggest that continued use of LET monitoring may be unjustified.

1. Kar R, Post A, John M, Rook A, Razavi M: An initial ex vivo evaluation of temperature profile and thermal injury formation on the epiesophageal surface during radiofrequency ablation . $J$ Cardiovasc Electrophysiol 2021 doi: 10.1111/jce.14911 PMID: 33476464.

2. Schoene K, Arya A, Grashoff F, Knopp H, Weber A, Lerche M, König S, Hilbert S, Kircher S, Bertagnolli L et al : Oesophageal Probe Evaluation in Radiofrequency Ablation of Atrial Fibrillation (OPERA): results from a prospective randomized trial . Europace2020 doi: 10.1093/europace/euaa209 PMID:

3. Chen S, Schmidt B, Seeger A, Bordignon S, Tohoku S, Willems F, Urbanek L, Throm C, Konstantinou A, Plank K et al :Catheter ablation of atrial fibrillation using ablation index-guided high power (50 W) for pulmonary vein isolation with or without esophageal temperature probe (the AI-HP ESO II) . Heart Rhythm 2020, 17 (11):1833-1840 doi: 10.1016/j.hrthm.2020.05.029 PMID: 32470628.

4. Meininghaus DG, Blembel K, Waniek C, Kruells-Muench J, Ernst H, Kleemann T, Geller JC: Temperature Monitoring and Temperature-driven irrigated Radiofrequency Energy Titration do not prevent thermally-induced Esophageal Lesions in Pulmonary Vein Isolation A randomized study controlled by esophagoscopy before and after catheter ablation. Heart Rhythm 2021 doi: 10.1016/j.hrthm.2021.02.003 PMID: 33561587.

5. Calkins H, Hindricks G, Cappato R, Kim YH, Saad EB, Aguinaga L, Akar JG, Badhwar V, Brugada J, Camm J et al : 2017 HRS/EHRA/ECAS/APHRS/SOLAECE expert consensus statement on catheter and surgical ablation of atrial fibrillation: Executive summary .Europace 2018, 20 (1):157-208 doi: 10.1093/europace/eux275 PMID: 29016841.

6. Leung LWM, Bajpai A, Zuberi Z, Li A, Norman M, Kaba RA, Akhtar Z, Evranos B, Gonna H, Harding I et al : Randomized comparison of oesophageal protection with a temperature control device: results of the IMPACT study . Europace 2020 doi: 10.1093/europace/euaa276 PMID: 33205201.

7. Clark B, Alvi N, Hanks J, Suprenant B: A Pilot Study of an Esophageal Cooling Device During Radiofrequency Ablation for Atrial Fibrillation . medRxiv 2020:2020.2001.2027.20019026 doi: 10.1101/2020.01.27.20019026 PMID:

8. Tschabrunn CM, Attalla S, Salas J, Frankel DS, Hyman MC, Simon E, Sharkoski T, Callans DJ, Supple GE, Nazarian S et al :Active esophageal cooling for the prevention of thermal injury during atrial fibrillation ablation: a randomized controlled pilot study . J Interv Card Electrophysiol 2021 doi: 10.1007/s10840-021-00960-w PMID: 33620619. 